Status of Issues in U.S. Edge-Plasma Research and Priority Topics for the Next Five Years

G. Bateman, C.-S. Chang, M. Fenstermacher, P. Guzdar, T. S. Hahm, S. Krasheninnikov, A. Kritz, T. D. Rognlien, D. Schnack, D. Schultz, P. Snyder, D. Stotler, J. Terry, M. Ulrickson, C. Bolton

March 21, 2005 
This document was prepared as an account of work sponsored by an agency of the United States Government. Neither the United States Government nor the University of California nor any of their employees, makes any warranty, express or implied, or assumes any legal liability or responsibility for the accuracy, completeness, or usefulness of any information, apparatus, product, or process disclosed, or represents that its use would not infringe privately owned rights. Reference herein to any specific commercial product, process, or service by trade name, trademark, manufacturer, or otherwise, does not necessarily constitute or imply its endorsement, recommendation, or favoring by the United States Government or the University of California. The views and opinions of authors expressed herein do not necessarily state or reflect those of the United States Government or the University of California, and shall not be used for advertising or product endorsement purposes.

This work was performed under the auspices of the U.S. Department of Energy by University of California, Lawrence Livermore National Laboratory under Contract W-7405-Eng-48. 


\title{
Status of Issues in U.S. Edge-Plasma Research and Priority Topics for the Next Five Years*
}

\author{
February 28, 2005
}

\section{Edge Coordinating Committee}

Glenn Bateman

Choong-Seock Chang

Max Fenstermacher

Parvez Guzdar (Secretary)

Taik Soo Hahm

Sergei Krasheninnikov

Arnold Kritz (Vice Chair)

Thomas Rognlien (Chair)

Dalton Schnack

David Schultz

Philip Snyder

Daren Stotler

James Terry

Michael Ulrickson

Curt Bolton (ex officio)
Lehigh University

New York University

Lawrence Livermore Nat.'1 Lab

University of Maryland

Princeton Plasma Physics Lab

Univ. of Calif., San Diego

Lehigh University

Lawrence Livermore Nat.'l Lab

Science Appl. Int'l. Corp.

Oak Ridge National Lab

General Atomics

Princeton Plasma Physics Lab

Mass. Institute of Technology

Sandia National Lab

U.S. Dept. of Energy, OFES

"Work was performed under the auspices of US DOE by the institutions above, including the following contract numbers: W-7405-ENG-48 at LLNL; DE-AC02-CHO3073 at PPPL, and DEAC05-00OR22725 at ORNL. 


\section{Executive Summary}

\section{Background and purpose}

The Edge Coordinating Committee (ECC) was formed in July 14-15, 2004 when OFES Theory Team invited 14 plasma researchers to a two-day meeting in Germantown, MD to discuss the state of edge-plasma research in the U.S. with a focus on theory and modeling (see http://www.mfescience.org/ecc/). At that time, OFES tasked the ECC with providing, in about a six month period, a report on the present status of key issues in this area together with a roadmap of what range of activities should be undertaken in the next five years to resolve these issues. This document is a response to that charge. Future edge-plasma research described here is assumed to fit into a budget constraint of a "flat budget," with some additional activities cited for budget increases of as much as $50 \%$.

To obtain some measure of the relative fraction of OFES Theory funding presently devoted to edge plasma research, the OFES Theory Team informally surveyed funded work they support in this area at National Labs, Universities, and industry. John Mandrekas reported to us that approximately $10 \%$ of the present budget goes to edgephysics areas at 10 institutions, for a total of $\sim \$ 2.5 \mathrm{M}$ each year. While not explicitly estimated, we note that there are also important edge plasma projects in the Plasma Technology program, especially related to plasma/material sputtering interactions, nearsurface plasma chemistry and transport, and impurity transport. Likewise, the Confinement Program has important efforts in interpreting edge-plasma data through modeling codes.

This initial status/roadmap work of the ECC is complimentary to the Fusion Energy Sciences Advisory Committee (FESAC) Panel on Program Priorities (see http://www.mfescience.org/fesac/). This Panel is charged with identifying major science and technology issues for all of controlled fusion and also with recommending campaigns to address these issues. The Panel has organized their report along six thematic areas, one of which, termed Plasma Boundary Interfaces, coincides directly with the interests of the ECC. Many of the ECC members have provided input to the FESAC Panel in the edge-plasma area, and an ECC member is on the Panel. The ECC is providing a more detailed examination of edge-plasma issues than is possible in the broad Panel charge and will also work to facilitate ongoing edge-plasma activities beyond the active term on the Panel. While the emphasis of the ECC is initially on theory and model development, there is a strong recognition that comparison and coordination with experiments and experimentalists are essential for meaningful progress; to this end, two members of the ECC are from the experimental community.

To gather data for the ECC task of a detailed summary and roadmap, edgecommunity input has been solicited at two meetings, the first being a workshop at General Atomics on Sept. 27-28, 2004, and the second a community meeting on Nov. 14, 2004 at the APS-DPP conference in Savannah, GA. Details of these meetings, topical summaries written by ECC members, and other information on the work of this committee can be found at the ECC website. A consolidation of this material forms the basis of this report. The focus here is on tokamak devices with magnetically diverted scrape-off-layer (SOL) separating closed and open magnetic field lines. However, there 
is substantial interest and work on 3D effects that thus begins the expansion of models and tools to include devices with intrinsic 3D variations such as stellarators.

\section{Edge regions and cross-cutting topics}

The edge-plasma region is defined as that from the top of the density and temperature pedestals, typically several $\mathrm{cm}$ inside the magnetic separatrix, to (and into) the material walls surrounding the plasma region. Thus, at the outer midplane, the edge region is $\sim 10 \mathrm{~cm}$ in radial extend as sketched in Fig. 1. This region includes steep gradients and strong interactions between plasma, neutrals, and photons, including hydrogen and impurities (atoms and molecules). Consequently, a multi-species description is required. The strong radial gradients result in rapid changes in plasma parameters that determine collisional effects and also provide free energy to drive various instabilities. For example, the higher plasma

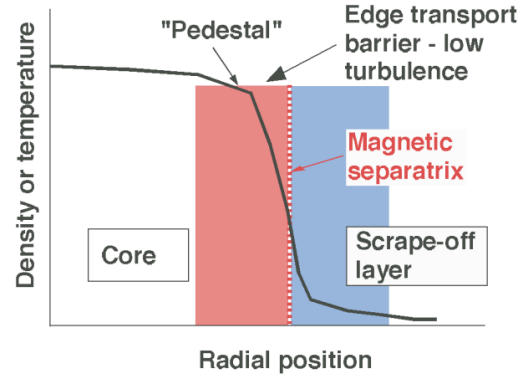

Fig. 1 Edge-region at midplane. temperature in the pedestal region typically results in a long mean-free-path collision regime that requires a kinetic description for rigorous description, whereas in the SOL, lower temperatures results in substantially larger collision rates, with the plasma in the divertor region typically being in the highly collisional regime owing to plasma density increase and further temperature decrease from ion recycling.

To help organize a summary of edge-plasma research, a 2D matrix is formed in Table 1, where the columns correspond to key physics areas often associated with subdomains of the edge region, while the rows correspond to tools and cross-cutting research. The presently described working model of this matrix is given with cryptic abbreviations that are more fully explained in the following summary.

Table 1. Key physics areas/issues and tools/activities for edge-plasma research.

\begin{tabular}{|c|c|c|c|c|}
\hline$\underline{\text { Issues/areas }}$ & $\begin{array}{l}\text { Pedestal } \\
\text { region: ELMs } \\
\text { \& L-H trans. }\end{array}$ & $\begin{array}{l}\text { SOL region: } \\
\text { transport, } \\
\text { wall fluxes }\end{array}$ & $\begin{array}{l}\text { Neutral and } \\
\text { photon } \\
\text { transport }\end{array}$ & $\begin{array}{l}\text { Plasma/mater.: } \\
\text { erosion } \& \\
\text { redeposition }\end{array}$ \\
\hline $\begin{array}{l}\text { Fundamental } \\
\text { models \& } \\
\text { equations }\end{array}$ & $\begin{array}{l}\text { Turb. \& coll. } \\
\text { transport; } E_{\mathrm{r}} ; \\
\text { kinetic effects }\end{array}$ & $\begin{array}{l}\text { Strong turb. - } \\
\mathrm{H}^{+} \text {\& impurity; } \\
\text { intermittence }\end{array}$ & $\begin{array}{l}\text { i-n and n-n } \\
\text { collisions, rad. } \\
\text { transp. }\end{array}$ & $\begin{array}{l}\text { Inter-atom pot.; } \\
\text { surface evol.; } \\
\text { sheaths; dust }\end{array}$ \\
\hline $\begin{array}{l}\text { Simulations \& } \\
\text { reduced/integr. } \\
\text { models }\end{array}$ & $\begin{array}{l}\text { MHD, 2-fluid, } \\
\text { MC ion \& 5- } \\
6 \text { D kinetic }\end{array}$ & $\begin{array}{l}\text { 3D MHD, 2- } \\
\text { fluid, \& 5-6D } \\
\text { kinetic codes }\end{array}$ & $\begin{array}{l}\text { Monte Carlo, } \\
\text { continuum, } \\
\text { fluid (2D, 3D) }\end{array}$ & $\begin{array}{l}\text { Molecular } \\
\text { dynamics; MC } \\
\text { ion; PIC }\end{array}$ \\
\hline $\begin{array}{l}\text { Atomic \& mol. } \\
\text { interaction } \\
\text { data }\end{array}$ & $\begin{array}{l}\text { Impurity rad., } \\
\text { ioniz., recomb. } \\
\text { rates }\end{array}$ & $\begin{array}{l}\text { Impurity \& } \\
\text { molecular } \\
\text { multi-step rates } \\
\end{array}$ & $\begin{array}{l}\text { Carb. Molec.; } \\
\text { chem., multi- } \\
\text { step, B-field }\end{array}$ & $\begin{array}{l}\text { Surface chem. } \\
\text { species \& rates }\end{array}$ \\
\hline $\begin{array}{l}\text { Verification \& } \\
\text { validation- } \\
\text { Eqns \& expt. }\end{array}$ & $\begin{array}{l}\text { Code-code; } \\
\text { US expt. \& } \\
\text { ITPA } \\
\text { databases }\end{array}$ & $\begin{array}{l}\text { Code-code; US } \\
\text { expt. \& ITPA } \\
\text { databases }\end{array}$ & $\begin{array}{l}\text { Code-code; US } \\
\text { expt. \& ITPA } \\
\text { databases }\end{array}$ & $\begin{array}{l}\text { Code-code; lab } \\
\& \text { fusion expt. }\end{array}$ \\
\hline
\end{tabular}




\section{Summaries of the 4 major issue areas and 4 tool areas in Table 1}

The issues/areas categories are represented by the columns in Table 1, and correspond to 3 spatial sub-domains of the pedestal, SOL, and walls; the neutral transport section is included as of interest in all regions. The section numbers in parenthesis below refer to the pertinent sections of the later, more detailed, full topic summaries. The ordering of the priority topics here is not meant to imply a ranking, and other issues of high importance are discussed in the longer topic summaries following this Executive Summary.

- The pedestal region (Secs. 1, 2, 3, 9)

- Issues: Located just inside the separatrix, it determines boundary conditions for the hot core plasma that provides strong levers for obtaining crucial improvements in energy confinement as well as high plasma pressure. High pedestal temperature and densities result from a transport barrier formed by suppression of plasma microturbulence in the L-H mode transition. The ultimate edge-plasma pressure gradient is believed set by MHD instabilities causing Edge-Localized Modes (ELMs) that periodically eject substantial quantities of hot plasma into the SOL.

- Status: While there are many theories for the L-H transition, there is no commonly accepted identification of the essential mechanisms and no routine simulations of transitions that are validated with experiment. The linear MHD theory of ELMs explains many aspects of experiments, but the nonlinear theory and simulations are at an early stage. Neoclassical ion transport has been simulated with Monte Carlo codes, but electron transport is not understood except by residual turbulence. The influence of kinetic and two-fluid effects on turbulence is only partially developed and validated, with most progress so far being in the fluid area. Radial electric field $\left(\mathrm{E}_{\mathrm{r}}\right)$ models from fluid and kinetic codes show deep $\mathrm{E}_{\mathrm{r}}$ wells inside the separatrix with similar magnitude to experiments, but detailed comparisons are limited. Likewise, various models show that neutral edge fueling can contribute substantially to the buildup of density in the pedestal.

- Priority items: Understand and predict pedestal width and height, including detailed generation mechanisms; nonlinear saturation and transport from ELMS; kinetic effects on turbulence and transport; understanding the L-H transition with full $\mathrm{E}_{\mathrm{f}}$ models; neoclassical loss and possible non-ambipolar current with residual $\mathrm{H}$-mode transport; role of residual turbulence on $\mathrm{E}_{\mathrm{r}}$ during H-mode; electron transport; neutral particle fueling.

- The SOL region (Secs. 4, 5, 9)

- Issues: The SOL plasma lies outside the separatrix and contacts the divertor plate and chamber walls along open magnetic field lines. Strong, intermittent plasma transport, both from microturbulence and ELMs, impact heat flux profiles on divertors and walls where peak values on divertors can be near operational limits. Relative fractions of plasma particle and heat flux to divertor plates compared to the nearby, less protected main chamber walls needs to be 
understood. Impurity transport through the SOL impacts core impurity levels (both sputtered-material impurities and fusion helium).

- Status: Basic ion-scale instability mechanisms identified, but not electron modes. The wavenumber and frequency ranges of strong SOL turbulence have also been observed in 2D and 3D simulations. Formation of large, local perturbations to the plasma density (blobs) is found in simulations, but there is limited understanding of detailed nonlinear development. The related analysis of blob transport mechanisms after formation has been studied in more detail, especially for the main hydrogen plasma species; the corresponding impurity transport has received only modest attention. Self-consistent profile evolution has begun, but is not yet routine. Mach probe measurements consistently find large parallel flows in the main chamber SOL that are usually not observed in transport modeling. Transport dynamics of large-scale ELMs in the SOL may be similar to blobs, but needs more development. Classical cross-field drifts and plasma currents, while studied in some detail, would benefit from more robust numerical algorithms. Kinetic parallel heat flux correction has some useable basic models, while general kinetic effects on turbulence are not well understood.

○ Priority items: Impurity transport with large-amplitude intermittency; selfconsistent profile evolution including neutrals and associated wall interactions; large parallel flows; ELM spatial and temporal transport to material surfaces; kinetic models of SOL turbulence and transport; fundamental understanding of blob formation coupled to propagation.

\section{- Neutral and photon transport (throughout edge region) (Secs. 4, 5, 6, 9)}

- Issues: Large populations of neutral atoms and molecules, and photons exist in regions of low to moderate electron temperatures $(\sim 1-100 \mathrm{eV})$ when there is strong plasma recycling, sputtering, and/or gas puffing. Transport of such neutral species controls hydrogen and impurity fueling as well as SOL energy loss via radiation. Both plasma-neutral and neutral-neutral collisions can be important. Impurities could have a direct impact on some instabilities. Helium ash must be pumped sufficiently to prevent fusion core dilution.

- Status: Hydrogen neutral transport understood from slower, more detailed Monte Carlo simulations and faster, less precise fluid models. Likewise, photon transport is beginning to be addressed for optically thick lines with Monte Carlo and multi-group models, as well as simpler escape-factor reductions. Impurity neutral transport, especially for hydrocarbon molecules is much less developed in the coupled hydrogen/impurity mode. Also, lack of detailed knowledge of reaction rates and general complexity of a large number of possible species keep progress slow. Neutrals are not yet routinely included in turbulent simulations, where they serve as a plasma particle source and could couple to the turbulence.

o Priority items: Improve efficiency of kinetic neutral models (Monte Carlo and hybrids) in high collisionality regimes and in coupling to plasma models; evaluate opacity effects for hydrogen; include hydrocarbon chemistry and coupling to the main plasma; add neutral models to turbulence simulations. 
- Plasma/Material interactions (Secs. 4, 5, 6, 9)

- Issues: Material composition, erosion, and redeposition determine the lifetime of plasma-facing components. Materials can store and re-emit hydrogen in response to varying plasma fluxes. In addition, such materials are sources of plasma impurities, including dust, and tritium deposition and transport are essential to understanding and controlling burning-plasma devices. The viability of carbon materials for future DT operation is in question because of experimental results showing large tritium co-deposition in remote locations.

- Status: Physical erosion and redeposition on single-component materials such as carbon seems reasonably well understood from Monte Carlo ion models. Carbon chemical sputtering is not well understood. Measured tritium migration to different areas of present-day DT tokamaks is far in excess of modeling results. Work is just beginning on mixed materials that are likely to occur in future devices. Simulations of ELM pulses incident on material surfaces have been preformed, but direct validation with tokamak data is quite limited. Initial simple models have been developed to characterize the transport of dust.

- Priority items: Understand and devise means to substantially reduce tritium codeposition with carbon; improve chemical sputtering models and data including mixed material effects (e.g., Be on C); improve and validate models for material response to ELMs; develop more detailed dust models, including formation, and assess impact on impurity production.

The rows of Table 1 correspond to developing tools, often-numerical simulation codes of varying complexity, and cross-cutting research activities as follows:

\section{- Fundamental models and equations (Secs. 7, 1,5)}

- Issues: Fundamental physics models are required for predictive understanding in all areas. While full configuration and velocity-space (6D) plasma kinetic equations are known, development of a 5D gyro-averaged kinetic equation valid for edge conditions (steep gradients, large fluctuations) will greatly facilitate progress. For Molecular Dynamics simulations, inter-atomic potentials are a fundamental requirement.

- Status: The plasma equations for the edge region are complicated by the existence of steep gradients and rapid spatial transition from short to long meanfree-path regimes. Consequently, kinetic equations obtained for orderings valid in the core are not directly applicable in the edge. Substantial progress has been made on fluid equations valid in the edge, and development of a 5D gyrokinetic edge formulation has begun. Extension to coupled kinetic impurities has not yet begun. Because of the absence of localization by the magnetic field, kinetic neutral and photon transport equations are inherently 6D. For interatomic potentials used in MD material sputtering, single-species (carbon) models are available, but the mixed materials needs development. 
- Priority items: Complete and verify a set of 5D gyro-kinetic plasma equations; develop inter-atomic potentials for sputtering of mixed materials.

\section{- Simulations and reduced/integrated models (Secs. 9, 8, 10)}

○ Issues: A wide variety of simulation codes are available for plasma, neutrals, and materials. These range from single-fluid (MHD) and two-fluid models in 2D and 3D for plasma transport and turbulence. Monte Carlo ion codes are used for kinetic effects, and 5D gyro-kinetic codes, both continuum and Particle-In-Cell (PIC), are under development. Likewise, neutral codes include fluid and Monte Carlo models, while PIC modeling is used for sheath physics. Molecular dynamic codes are used for sputtering calculations. Validated reduced models that capture the essential physics are needed to provide descriptions for integrated models of the edge, and efficient numerical coupling schemes must be developed.

○ Status: Fluid simulation models are used routinely for 2D transport of hydrogen and impurity plasmas, and a few codes exist for 3D transport in nonaxisymmetric devices such as stellarators. Turbulence in hydrogen edge plasmas is described by various fluid codes ranging from 2D slab models to 3D codes that can include the geometrical complexities of toroidal devices. Underlying fluid models range from the single-fluid MHD equations to the twofluid equations, with a new set of more complete two-fluid equations published in the last year. Numerical algorithms include finite difference (or volume), finite elements and particle-based schemes, which use either semi-implicit or fully implicit time integration. Use of parallel computers is fairly standard, but likely not optimal. Kinetic simulations are typically of reduced spatial dimension and include continuum and particle-based methods (Monte Carlo or PIC). MD codes use particle-like techniques. Numerous reduced models of various components are used in the codes with varying degrees of verification that are able to model aspects of interacting physical systems such as plasma, neutrals, and radiation. Some core transport codes have reduced models for the pedestal region, including the stability and impact of ELMs.

○ Priority items: Develop 4D (transport) and 5D (turbulence) gyro-kinetic codes; implement improved edge fluid models for transport and turbulence; improve robustness and efficiency of coupling different physics models for integrated studies; utilize advanced computational techniques to enhance usage of largescale computing.

\section{- Atomic and molecular interaction data (Secs. 6, 4, 5)}

○ Issues: The partially ionized edge plasma requires accurate models of atomic and molecular interaction with electrons, ions, and neutrals. This includes electron-impact ionization, dissociation, and recombination with association energy loss via escaping photons. Ion-neutral and neutral-neutral collisions are also important. Molecules introduce vibrational states that complicate the analysis. Surface chemistry processes and rates are also required.

- Status: Hydrogen processes and rates are fairly well understood, but those for impurities and especially molecules have substantial gaps in knowledge. 
Hydrocarbon species are particularly numerous and their interaction rates are poorly understood at best. Likewise, confidence in surface chemistry models is weak, especially for evolving surfaces.

- Priority items: Organize and improve rates for hydrocarbons; identify important surface chemistry and determine an effective approach for modeling; assess impact of metastable species.

\section{- Verification and validation of models (Secs. 10, 11)}

- Issues: All models should be verified and validated to the extent practical. "Verification" entails establishing that the code accurately solves the mathematical equations underlying the model. "Validation" consists of an assessment of the extent to which that model is a plausible description of physical reality as determined by comparison with experimental data. Owing to the number of different physical processes in the edge region, fully selfconsistent validation is a challenging problem. This activity provides the essential link to the reason for theory and simulation research, namely, the understanding of experimental results that guides further device operation and new device design.

- Status: Although most numerical edge models have been verified to some extent, the level of rigor involved has for the most part been less than that found in other fields such as computational fluid dynamics. A number of fruitful code-code comparisons have been undertaken; such efforts can contribute to verification. Considerable effort has been made to compare models with experimental data. For some models, there are key elements that need to be calibrated or fit, such as anomalous diffusion coefficients in plasma transport codes. Such "validation" tests the consistency of numerous other physics components of the model subject to the few fit parameters, which can be thus viewed as an "interpretive" simulations used to estimate the size of unknown processes and is not truly predictive. In this example, turbulence codes are being used to provide more fundamental radial transport coefficients that are beginning to replace the fitting procedure. Thus, more fundamental "validation" follows as the models mature. The code-experiment comparisons tend to be either isolated detailed comparisons of single discharges, providing insight into the physical processes in that discharge, or less constrained simulations to elucidate scalings of parameters under varying experimental conditions.

○ Priority items: Develop more rigorous verification procedures by establishing standard test cases and communication of verification techniques; improve interaction between modelers and experimentalists to begin the development of well-planned validation experiments and corresponding simulations. Some key items requiring understanding and validation are pedestal structure, tritium migration, dynamics of the ELM crash, particle fueling, and SOL transport/turbulence. 


\section{Topic Summaries Prepared by Topical Subgroups}

The material in these sections was developed by small subgroups of mostly ECC members whose edge-plasma research has been focused in these different areas. In addition, the groups drew upon information from colleagues, and from the discussions and presentation at the two community meetings held in September 2004 at General Atomics in San Diego, CA, and in November 2004 at the APS-DPP meeting in Savannah, GA. Each topical coordinator then took the responsibility for distilling this material into following summaries. The Executive Summary section above gives an overview of these more detailed descriptions.

\section{Stability and Dynamics of Edge Localized Modes (coordinated by P.B. Snyder)}

Edge Localized Modes (ELMs) are repetitive perturbations of the edge barrier region, which transport particles, and usually heat, across the separatrix and into the open field line region. ELM physics is of considerable importance, particularly for Next Step fusion devices such as ITER, for two primary reasons: The first is that the instabilities responsible for ELMs provide an effective constraint on the maximum achievable pedestal height for given profile shapes. The pedestal height in turn has a substantial impact on core confinement and therefore overall fusion performance. The second issue is that the localized heat and particle pulses associated with the ELM events themselves, while largely benign in present devices, have the potential to significantly impact plasmafacing material lifetimes in Next Step devices.

An understanding of both the linear physics that determines ELM onset conditions, and the nonlinear physics that determines the evolution of the modes, including the heat, particle, and momentum losses from the core plasma, and heat and particle deposition on material surfaces, is needed to fully address these issues and provide quantitative projections of optimized pedestal height and plasma performance in steady state regimes with tolerable or no ELMs.

\subsection{Linear Physics Status}

Substantial recent progress in pedestal stability theory, computation, and high resolution measurement of pedestal profiles has led to a broadly held conclusion that many types of ELMs can be attributed to MHD instabilities driven by the sharp pressure gradients and consequent large bootstrap current in the edge barrier region. These instabilities, typically most unstable at intermediate wavelengths $(3<\mathrm{n}<40)$, provide a quantitative constraint on the pedestal height as a function of equilibrium parameters such as plasma shape, collisionality, pedestal width, current, and magnetic field. Recently developed computational tools have allowed a number of successful comparisons to observed ELM onset time and characteristics.

These comparisons have been done primarily using static ideal MHD physics models, though efforts have also been made to incorporate the effects of diamagnetic stabilization, rotation, resistivity and other two fluid physics. Additional effort is needed to perform linear edge stability calculations using comprehensive physics models. Such physics models exist for highly collisional regimes, but require development and 
extension to less collisional plasmas found near the top of the pedestal, particularly in Next Step devices. Alternatively, weakly-collisional gyrokinetic models can be extended to deal with the high collisionality near the bottom of the pedestal, as well as large $\rho / L$, non-local strongly shaped geometry and full electromagnetic physics. In addition, very high resolution edge profile measurements, including where possible direct measurement of the current profile is important for the rigorous testing of theoretical models and evaluation of the impact of non-ideal effects.

Previous studies have focused primarily on Type I (both large and small) and to a lesser extent Type II ELMs. The physics of other regimes such as Type III, EDA, QH etc is less well understood. Furthermore, a number of ELM control techniques have been developed, both theoretically motivated and empirical, which introduce additional features that must be incorporated into physics models. For example, the use of nonaxisymmetric magnetic fields to modify ELM behavior creates a need for 3D computation even in linear studies. Pellet triggering of ELMs also introduces nonuniformity and significant new physics. Strong $\mathrm{E}_{\mathrm{r}}$ effects, and possibly also kinetic orbit effects may impact the physics of low collisionality QH mode plasmas.

\subsection{Nonlinear Physics Status}

Nonlinear studies are needed to quantify ELM heat and particle losses from the core plasma and their deposition on material surfaces. However, nonlinear simulations of the edge region introduce significant challenges. The range of spatio-temporal scales one wishes to study is in general very large and not separable, in part due to the substantial variation in equilibrium quantities from the hot pedestal top to the cold scrape-off-layer. Fluctuating quantities can be large compared to time averaged values, and usual perturbative techniques cannot generally be used. In general, one requires a physics model that can treat both the relatively collisionless plasma near the pedestal top and the highly collisional plasma in the SOL. Because of the wide range of scales, nonlinear simulations of the full ELM crash event, even in 3 dimensions, will require efficient numerical methods and computational resources beyond those presently available.

A semi-analytic nonlinear ballooning theory has been developed in the high-n limit, predicting explosive mode growth in the early nonlinear phase. Existing largescale 3D numerical studies have generally reduced the range of scales by initializing with an unstable equilibrium. 3D simulations of the early phase of the ELM crash have been carried out both with reduced Braginskii two fluid physics, and with single fluid extended MHD models. Formation of filaments resembling observations has been seen in nonlinear reduced Braginskii simulations.

However, 3D simulations have so far been successful only in studies of the early nonlinear phase. Longer simulations, using higher resolution and/or adaptive techniques, which allow the magnetic equilibrium to evolve, along with realistic wall and divertor plate boundary conditions, are needed to follow the ELM pulse all the way to material surfaces and study the collapse of the pedestal and relaxation of edge current and rotation profiles. Longer duration and full turbulence scale physics is needed to allow initialization with a stable equilibrium, and slow transport timescale evolution across the stability boundary, followed by the ELM collapse. Higher spatial resolution is needed to treat both the relevant range of mode numbers, and full torus $(\Delta n=1)$. Furthermore, either kinetic extensions of 3D fluid models, or development and implementation of appropriate 
electromagnetic 5D or 6D kinetic models, are needed for evaluation of the impact of kinetic effects on ELM dynamics.

In summary, substantial progress has been made in understanding the physics of ELMs, but many challenges remain, particularly in the development of realistic nonlinear theories and simulations over the full relevant range of scales.

\subsection{Linear Physics Five Year Goals}

- Extensive comparisons of linear stability to ELM observations across all major tokamaks

- Comprehensive evaluation of the impact of effects beyond static ideal MHD (diamagnetic stabilization, rotation, resistivity...) in the collisional fluid limit

- Development and some implementation of kinetic and kinetic-fluid formulations for linear stability in the collisionless and transition regimes

- Studies of 3D equilibrium configurations such as "stochastic edge"

- Goal is to develop a clear physics understanding of ELM onset conditions in wide range of regimes

\subsection{Nonlinear Physics Five Year Goals}

- Development and implementation of improved fluid formulations in 3D codes (eg Simakov-Catto, kinetic-fluid...)

- Successful use of 2-fluid formulations in both field-aligned (eg BOUT) and unaligned (eg NIMROD, M3D) ELM simulations

- Extend range of scales treated in 2 fluid simulations

○ Toroidal: for some ELM types, full torus up to gyroradius

- Radial: extend boundary to material wall (with proper BC), and further into core

- Time: Extend simulations beyond early ELM phase (higher resolution, adaptive grid...). Include sources/sinks and drive pedestal slowly across stability boundary.

- Improve numerics of existing codes, implement on next generation machines

- Develop kinetic formulations appropriate for large $\rho / \mathrm{L}$, collisionless to collisional transition

- Some limited-scale studies with 5D (and possibly 6D) codes

Note that doing the above, particularly exploring the stated range of spatiotemporal scales, even in a 3D code, requires computational resources well beyond those presently available. In many cases progress will have to be made piecemeal, focusing on understanding the physics occurring within a more limited range of scales.

To be successful, this work will need to be carried out in close collaboration with experiment (possibly with new fast diagnostics in the edge), inter-ELM transport and source physics, code $\mathrm{V} \& \mathrm{~V}$, and integrated modeling. 


\subsection{Additional Progress Possible with a Strong Edge Initiative}

Existing efforts are strongly constrained by limited human and CPU resources. Existing ELM simulation efforts already go beyond resources available at NERSC for this work. A new initiative could substantially increase collaboration, cross comparison, and V\&V of codes, and allow development of essentially new code(s) with state-of-theart numerics and physics.

With a strong initiative increasing the number of involved scientists and the availability of computational resources, full simulation of the ELM crash, pedestal collapse and heat and particle loads on material surfaces should be possible within a 3D code framework. Development of appropriate (5 or 6D) kinetic formulations, and their implementation in limited scale simulations, such as local evolution of fast electron energy during ELM bursts, should also be possible. Progress in understanding pedestal constraints and the ELM crash should be substantial enough to allow improvements both in identification of optimum operational regimes and in active ELM control.

\section{Edge pedestal buildup (coordinated by C.S. Chang)}

After an edge transport barrier formation at an L-H transition, the plasma edge gets into a quiescent state and a plasma pedestal builds up just inside the separatrix. The profile shape of a density pedestal is sharper and shows a more consistent behavior than the ion and electron temperature pedestals. The density pedestal width is typically narrower than an ordinary thermal banana width measured at the pedestal top. The electron and ion temperature pedestals usually penetrate further into the core plasma, with the ion temperature pedestal sometimes being much milder extending into the scrape-off plasma. Inner edges of the pedestals are often observed to penetrate further into the main plasma as the pedestal height grows in time. Except perhaps at low densities, the density pedestal initially rises more quickly than the temperature pedestal.

Pedestal buildup is usually interrupted by repetitive ELM crashes, which can provide a time averaged saturation of pedestal height. Even with the repetitive ELM crashes, the confinement enhancement in the H-mode over an L-mode is substantial. The anticipation is that it will be an attractive scenario to operate a tokamak reactor in a proper ELMing H-mode. In some cases, however, the pedestal can build up without an ELM crash, in which case the plasma is generally not in steady state and the improved impurity confinement by the transport barrier induces too much impurity radiation to sustain the H-mode. There is yet another severe problem foreseen in an ELM free buildup of pedestal in a tokamak reactor, even if the impurity accumulation problem can be avoided. The improved particle confinement may result in an intolerable accumulation of Helium ash particles.

Among several physical quantities to be predicted in the edge pedestal buildup, the pedestal width and height may be the most urgent one. Since the ELM criterion may generally be a constraint on the pedestal height at a given radial width and structure, the pedestal height may be determined by the pedestal width and structure. As for the L-H transition, there can be different physical explanations for the pedestal growth, width and structure, ranging from the self-organization of plasma turbulence, to neoclassical ion transport with neutral penetration, to some hybrid model of these phenomena. However, basic research in all these areas is far from completion. We have to develop these 
researches further before we can truly integrate them for prediction of the H-mode pedestal structure and buildup process with confidence.

Comprehensive edge pedestal physics may cover multiple collisionality with a wider range of spatiotemporal scale-lengths than any core physics has been dealing with. This will be part of a grand challenge which the pedestal buildup physics faces. The ions in the pedestal region in modern large tokamaks and future reactors are likely to be in a weakly collisional, kinetic regime, surrounded by collisional scrape-off plasma where the incoming neutral dynamics is determined. The fact that the pedestal width is smaller than the ordinary thermal banana width insures the importance of ion orbit dynamics in a selfconsistent radial electric field across the pedestal and separatrix. Some of these orbits, especially those passing through the X-point region, intersect the first wall and may play an important role in pedestal buildup physics. The ion orbit dynamics in a strong radial gradient may induce a different bootstrap current from that predicted by a weak gradient theory. The neoclassical orbit dynamics may change the electric field in less than an ionion collision time, closely relating its physics to that of zonal flow. These physical phenomena are beyond fluid description. Thus, a fully kinetic study is an essential long term goal in the research of pedestal buildup physics, while improved fluid studies with properly reduced equations from kinetic equations may achieve short term goals. Due to the strong gradient, which defies the conventional analytic ordering, and the existence of the separatrix (and X-point) where the safety factor $\mathrm{q}$ is infinite, even a basic analytic neoclassical analysis has been extremely difficult. The existence of neutral particles makes the analysis even harder.

Thanks to recent development of powerful modern computers, though, large-scale numerical simulations have started and shown promising progresses. Most of future progress in this area is expected to be made by large-scale numerical simulations. The kinetic pedestal simulations should focus on the short time scale pedestal buildup between ELM crashes (on the order of 10 milliseconds or less). These codes should describe the ion neoclassical, neutral, and turbulence transports in the presence of impurity particles including self-consistent $\mathrm{E}_{\mathrm{r}}$. Development of both continuum and discrete kinetic methods has been suggested. Electron neoclassical transport is too slow to influence the pedestal growth in this time scale. The importance of neutral penetration and ionization calls for a proper modeling code for scrape-off plasmas and plasma wall interaction.

There may be some residual turbulence in the H-mode layer, which could influence the pedestal buildup, especially in the electron temperature profile. Evidence for an elevated electron heat transport is often seen from a buildup of electron temperature pedestal during an ELM cycle, which is not possible in a quiescent plasma due to the negligibly slow neoclassical electron transport as mentioned earlier. Electromagnetic gyrokinetic turbulence codes need to be developed to study this problem. The gyrokinetic codes to be used for pedestal buildup will have to be valid in the steep pedestal gradients with the gradient scale length coming down to a few ion gyroradii, under X-point geometry, and in the presence of neutrals (these requirements may be more stringent than for an L-H transition code).

The present gyrokinetic equations may need much further improvements to satisfy these severe requirements. However, considering that at least a few years are needed to develop such a code, the code work must not wait for the completion of the gyrokinetic 
equations. Another issue to be resolved in relation to the electron heat transport and other pedestal phenomena is the role of stochastic field lines near the separatrix. The stochastic field lines may effectively move the open field lines to inside the separatrix surface and induce electron heat transport in the pedestal. In the experimental side, in addition to measuring more refined spatio-temporal pedestal structures, measuring poloidal distribution of plasma flow and neutral density profiles are in high demand for a more proper support of and comparison with the development of pedestal buildup theory.

\subsection{Five Year Priority Issues}

In order to understand the pedestal shape and width, buildup dynamics, ExB shearing rate, plasma current change, and others, we need to resolve numerous issues including

\section{At present funding level:}

- Neoclassical ion transport in the pedestal region with neutral recycling and NBI:

The aim is in the self-consistent, time dependent simulation of pedestal buildup in a quiescent edge, evaluating the profile evolution of density, electrostatic potential, ion temperature, ion bootstrap current, plasma flow, plasma viscosity and radial momentum transport. The effort should include effect from a single species impurity ions, neutral recycling and transport, neutral beam injection, RF heating, nonlinear Coulomb collision operator, and poloidal variation of electrostatic potential.

- Residual turbulence and error field:

Ion and electron residual turbulences need to be studied under an ExB shearing rate predicted by the neoclassical theory or by the turbulence self-regulation. Capability to predict suppression of ion turbulence transport in H-mode needs to be established from first principles. A particular emphasis should be placed on the electron turbulence transport. Relevance of the non-axisymmetric perturbation in the equilibrium magnetic field (error field) should be verified.

- Pedestal scaling law:

Based upon the above advances, we should build a pedestal width scaling law, predicting the width and structure of density, electron temperature, and ion temperature pedestals..

\section{With a 50\% funding increase:}

- A self-consistent particle fueling physics:

Pedestal physics can be dependent upon poloidal fueling distribution and fueling dynamics. A more complete kinetic ion-electron-neutral transport modeling in the scrape-off region, including a proper neutral wall-recycling analysis, needs to be completed.

- Impurity effect on pedestal physics:

Impurity can have a significant effect on the electric field profile in the pedestal region, affecting the pedestal shape and width. A proper scrape-off impurity transport and wall recycling modeling is required. 
- Auxiliary heating effect on pedestal physics:

NBI and RF heating may also contribute to pedestal growth and structure. A proper NBI and RF heating model needs to be included for a more confident study of pedestal physics.

- Combined neoclassical, residual turbulence, and scrape-off modeling:

By combining the neoclassical, residual turbulence and scrape-off layer modeling into a single code, we can have a more complete and consistent description of the pedestal buildup physics.

\section{L-mode to H-mode confinement transition (coordinated by P.N. Guzdar)}

Understanding the physics of the L-H transition in tokamak edge plasmas is an important area of research since it will lead to the design of an efficient fusion reactor. Thus the study of this spontaneous, self-organized, good-confined mode in tokamak plasmas has been an active area of experimental, theoretical, computational and integrated modeling research for the last decade. The fundamental paradigm that qualitatively explains the improved confinement that has gained widespread acceptance, is based on the creation of an edge boundary layer inside the last closed flux surface, in which a strongly sheared flow suppresses turbulent fluctuations responsible for the poorly-confined L mode and causes the plasma to transition into the well-confined $\mathrm{H}$ mode. An alternate scenario advocates strong suppression of the turbulent fluctuation due to finite beta effects without invoking shear flow stabilization. Although a convincing causal relationship between the generation of shear flow and improved confinement leading to the transition has not been established, the generation of the observed sheared flow and its consequence on transport has been one of the major areas of theoretical, computational and modeling research for the last two decades.

There have been a variety of mechanisms identified that could generate sheared flow. The basic mechanisms for the generation of this flow and an associated radial electric field require either toroidal effects, such as ion-orbit loss and poloidally asymmetric transport driven Stringer spin-up, or the action of generalized Reynold's stress of the turbulent waves responsible for the anomalous transport, which become unstable to the generation of the flow. Some of these models then derive criteria for the onset of the L-H transition. The next class of studies that have been undertaken has focused on using first principles derived or empirical shear-flow modified transport coefficients in 1D or 2D transport codes to understand the temporal and spatial dynamics of the bifurcation leading to the transition and subsequent (Edge Localized Modes) ELMy behavior. Finally at the most sophisticated level, fully three-dimensional fluid simulation codes which provide first principle modeling of the anomalous edge turbulence and transport and their interplay with self-generated or imposed shear flows have been developed. Studies at all these various levels of sophistication have in fact helped and instigated diagnostic development for the edge plasma. High-resolution profile measurement diagnostics for the density, temperature as well as flows have been implemented on major devices since the scale-length of the shear boundary layer is very narrow compared to the size of the discharge. By comparing the inferred shearing rate in 
specific discharges to the growth rate of characteristic instabilities (like the ion temperature gradient modes) albeit for the internal transport barriers, the fundamental paradigm of flow shear suppression of the turbulence has been well established and documented. Diagnostics for studying the spatial and temporal characteristics of the fluctuation in the $\mathrm{L}$ and $\mathrm{H}$ mode phases have been developed and detailed comparisons of the structures and spectral characteristics with the three-dimensional simulation codes have proven to be very valuable. Thus there has been significant all-round progress in theory, computation, modeling and experiments.

However many of the theories have limited predictive capability due to, for instance, their dependence on anomalous transport coefficients which are unknown. Thus comparison with observations is not possible. If the zonal flow driven by plasma turbulence is a viable mechanism for the transition, there is no direct experimental demonstration of this key physics prediction. The three-dimensional fluid codes have been developed in isolation without proper benchmarking between them. Although some of the codes only deal with the region within the Last Closed Flux Surface (LCFS) and others incorporate the Scrape Off Layer (SOL), the common elements of these codes need to be benchmarked. Also the use of fluid equations is suspect for predicting reliably the pressure pedestals in the $\mathrm{H}$ mode since their validity is questionable in the region of the top of the pedestal. Integrated modeling has many empirical inputs, which limit their predictive capability. Thus the edge program lacks focus and coordination.

The primary goal is to have a well-coordinated edge program which will focus on developing competing theory, simulations and modeling driven by observations, some of which focus on testing key physics elements, so as to have the desirable predictability for better understanding of present-day machine performance and potential for making reliable predictions for the next generation of burning plasmas in tokamaks. Thus the basic recommendations, expectations and goals for the next five year period are

\section{at flat funding level:}

- Self-consistent simulations of the L-H transition using existing edge turbulence and transport fluid codes, which have been suitably benchmarked.

- Detailed comparison of these simulation results with experimental observations to determine the relevance of fluid models and key physics present in the current codes.

- Firming up theory models to have predictive capabilities for comparison with both observations and simulation results on key physics issues advocated by the theories.

- Derivation of new set of equations, kinetic and extended MHD with "collisionless" physics valid for describing the edge plasma with strongly varying equilibrium profiles

- Seamless integration of codes dealing with regions from the top of the pedestal needs to be undertaken 
- Integrated modeling codes incorporate theory and simulation based results to have genuine predictive capability

\section{With a fifty percent increase:}

- A new generation of simulation codes, kinetic and extended MHD with "collisionless" physics based on the equations derived in item(4) with strong community involvement and support.

- Integrate these turbulence codes with transport codes.

- Elucidate new key physics obtained from such codes to stimulate new theories or modify existing ones and compare new features with focused campaigns on current machines..

The use of current fluid codes and subsequent development of fully threedimensional turbulence and transport code, which can simulate a self-consistent L-H transition, is a very pressing issue. This requires coupling between turbulence and selfconsistent evolution of the profiles for the plasma density temperature and flows i.e. the slowly evolving background equilibria. This level of modeling will render the most detailed information on both the micro (spatio-temporal characteristics of the turbulence) and macro level (plasma profiles) for comparison with observations. It will help in selecting and reassessing the many physics-based theoretical models that have been developed. Furthermore by parametrizing the anomalous turbulence they will provide input for the integrated modeling initiative. However the scope of the present-day codes and the new generation codes go beyond addressing the physics of the basic L-H transition and its scaling with plasma parameters. This will also provide information of the post transition dynamics of the ELMs and the different regimes of ELM behavior and even transport induced disruptive density limits. Furthermore comparison of results from present-day fluid codes with the next generation of kinetic codes will clearly address the issue of the role of collisionless physics in the transition.

\section{L-mode turbulence, transport, and the density limit (coordinated by \\ S.I. Krasheninnikov)}

The behavior of plasma turbulence and associated transport in the edge region is generally believed to be a crucial component in the controlling core energy confinement, resulting in either low confinement (L-mode) or high confinement (H-mode). The Lmode edge is characterized by strong low-frequency plasma instabilities and turbulence with relatively short wavelengths; the toroidal wave mode numbers are typically greater than about 40. Modes having toroidal mode numbers less than 40 are typically associated with ELMs described in another section. It is important to understand the L-mode regime for various reasons; e.g., (1) L-mode can dominate start-up periods of plasma discharges that can be very long for large devices, yielding large particle and power fluxes to material surfaces, (2), optimized suppression control of turbulence for the H-mode requires understanding of specific edge turbulence mechanisms, and (3), the 
performance-degrading density limit observed in all tokamak devices is associated with the return of L-mode levels of edge turbulence.

There are a number of mechanisms for plasma instability has been identified in the edge region where steep plasma gradients and sheared flows can drive them to high levels, although there is not yet a full self-consistent picture of edge plasma turbulence. These mechanisms include the following: the electron drift wave instability, X-point resistive mode, instability caused by parallel shear of ExB drift velocity, resistive ballooning instability, and instabilities on open magnetic field lines driven by synergetic effects of plasma cross-field inhomogeneity and plasma contact with material surface through the plasma sheath (so-called sheath-driven modes). The special features of divertor geometry with a magnetic separatrix give rise to the $\mathrm{X}$-point resistive mode. Plasma contact with material surfaces on open field lines results in both the emergence of modes that do not exist in the core region (such as $\nabla_{\perp} T_{e}$ modes), and significant modification of the modes that can arise on closed flux surfaces (for example, associated with ballooning effects). Linear stability codes and nonlinear fluid turbulence codes predict the existence of such instabilities and turbulence in the edge region.

Understanding of the special characteristics of edge turbulence and the associated plasma transport has evolved substantially in both experimental and theoretical areas. Fast imaging cameras, reciprocating Langmuir probes, Beam-Emission Spectroscopy, and gas-puff imaging often find turbulent fluctuations in the scrape-off layer (SOL) that are of the order of temporally averaged values. Furthermore, the plasma perturbations are found to propagate radially at velocities in the range of $100-1000 \mathrm{~m} / \mathrm{s}$ or more as filamentary, field-aligned structures especially in the outer SOL. The plasma filaments have cross-B-field size of one to several $\mathrm{cm}$. During the same discharges, there is often strong particle recycling and power deposition near the divertor strike points as observed in imaging cameras, IRTV thermal images of the divertor surface, Thomson scattering, and Langmuir probes. Thus, both strong radial transport and parallel transport can be important, with the strong, but intermittent behavior of the radial transport being revealed by various diagnostics. Such radial transport can occur both in L-mode and sometimes between ELMs in H-mode. Furthermore, similar filamentation can be seen in the detailed signals from an ELM, especially as it propagates into the outer SOL. Similar features of plasma turbulence have been observed in stellarators and linear-plasma devices, which suggests that the intermittent, filamentary plasma structures represent a fundamental property of cross-B-field plasma transport.

The rapid outward motion and other characteristics of the plasma filaments, or "blobs," have been explained by models invoking ion and electron drift motions that polarize these localized plasma structures. The origin of the "blobs" is the plasma turbulence mentioned above. Reduced two-dimensional plasma simulations of initialized "blobs" have also been used to understand and confirm analytic models. For whole-edge transport modeling, the radial transport can be described more naturally as a strong outward convection, but sometimes it is possible to use a strongly increasing radial diffusion coefficient as one moves toward the outer wall. It has been shown that "blobs" can play an important role in the particle and energy transport in the SOL region. The contribution of non-diffusive flux associated with transport of blobs to the total particle flux in the far SOL can attained 70-90\% for some transport simulations. 
Another aspect of experimental fluctuation data from diverted tokamaks that corresponds to theoretical understanding is the weaker edge plasma turbulence and transport on high-field side of the torus compared to the low-field side while operating in single-null configuration. In double-null configuration, which topologically separates high- and low-field sides of the SOL, plasma turbulence and transport on high-field side decrease even more. These observations indicate that ballooning effects associated with the destabilizing magnetic curvature on the low-field side of the torus play a very important role in edge plasma turbulence and transport, as expected from theory and observed in turbulence simulations. Although details depend on particular magnetic geometry used in the each turbulence code, it appears that all agree that decreasing edge plasma temperature results in the turbulence becoming more and dominated with the resistive ballooning mechanism. Simultaneously, transport becomes more and more convective rather than diffusive with coherent structures (blobs) rapidly propagating toward the wall. These simulation results are in reasonable qualitative agreement with experimental observations.

Numerical modeling also show that after some level, further decrease of edge plasma temperature (often associated with an increase in plasma density) causes a very strong enhancement of plasma turbulence and transport, which can be interpreted as an onset of so-called density limit. However, there is no clear physical picture of why the resistive ballooning mode becomes so strong and dominant at low plasma temperatures, and no theory-based scaling for the density limit exists. Moreover, the experimental picture of the density limit is more complex than current numerical simulations, although it is observed to yield high turbulence levels. In practice, the density limit is often accompanied by the formation of the MARFE and divertor detachment phenomena, including impact of radiation effects and plasma-neutral interactions. Typically such effects are not accounted for directly in 3D turbulence modeling, although uncoupled transport modeling with impurities, with transport coefficients guided by turbulence simulations, show this effect. Also, the enhancement of convective-like cross field plasma transport and end plate detachment at enhanced plasma density and/or reduces temperature are also observed in linear devices where there are no ballooning (or curvature) effects. Nevertheless, it is plausible that neutral wind and/or centrifugal forces substitute for the destabilizing magnetic curvature on the low-field side of a torus. In spite of the various limitations of the models, the simulation trends of increased (decreased) plasma turbulence and transport at increased plasma density (temperature) is encouraging.

\section{Plasma materials interactions (coordinated by M.A. Ulrickson)}

The loss plasma from the core primarily moves along the open field lines in the SOL to the divertor. A major accomplishment of experiments and modeling has been that at high core plasma density, the SOL plasma can be well isolated (or partially detached) from the divertor - this is the essence of the ITER design. While most of the plasma interactions with the wall are at the SOL-divertor interface, there can also be radial losses in the SOL that result in less severe, but significant interactions with the first wall. Bursts of heat and particles due to Edge Localized Modes (ELMs) are transported through the SOL and cause large, repetitive loads on the divertor and first wall. All of these plasma-materials 
interactions (PMI) involve heat and particle fluxes on the first wall and divertor. Examples of PMI include collisions of ions with the wall causing sputtering, chemical processes that erode the wall, and the deposition of impurities and plasma particles in the walls. Plasma facing components (PFCs) must absorb large heat and particle fluxes while minimizing erosion and plasma contamination.

Plasma bombardment of plasma-facing surfaces causes erosion of the plasma facing material. The eroded material acts as an impurity in the hydrogen plasma, and may be transported to the core plasma where it adversely affects fusion performance, locally re-deposited, or recycled causing enhanced erosion. Concurrently, the hydrogen is ion implanted into the surface, which can act as a larger reservoir for the hydrogen fuel than the plasma volume itself. At locations where the impurities build up deposition layers, the radioactive tritium hydrogen fuel is effectively trapped and accumulates at a high rate; tritium inventory in deposit layers is a major issue for burning plasmas. Conditioning of the plasma facing surface is often accomplished by glow discharge cleaning between pulses or by coating the wall, but conditioning time is usually much longer than operating time. Long pulse operation is virtually unexplored with regard to changes in wall conditions or material damage due to bombardment. Effective methods for tritium inventory control and understanding of impurity transport are essential for long pulse burning plasma operation.

A basic aspect of plasma contact with a solid surface is the formation of a sheath, but the ratio of power to particle flux densities and the sheath heat transmission coefficient, $\gamma_{\text {sh }}$, whose experimental values can differ greatly from the Langmuir-Bohm value of $\sim 7$, being often only $\sim 1$ near the outer strike point, the regions of most intense plasma heat conduction to solid surfaces are not understood.

It has been suggested that dust particles might be a source of plasma impurities in the early stages of a tokamak discharge. The presence of significant amounts of dust has been observed in the chambers of many fusion devices. The impact of dust particles on the performance of current fusion devices has still not been adequately understood. The existence of dust particles in burning plasma devices may also pose a significant safety threat due to the accumulation of tritium and radioactive materials. Therefore, the whole spectrum of dust associated issues including dust formation, transport, and dust-plasma interactions for fusion plasma conditions should be carefully studied on current machines.

\subsection{Fundamental Experiments}

Erosion yields for a wide variety of materials (including some liquids) and incident species have been measured in monoenergetic beam experiments and plasma simulators. Chemical erosion is studied using atom exposure at elevated temperatures and monoenergetic beams. Synergistic effects like radiation induced sublimation and ad-atom evaporation experiments are performed using beams at elevated temperatures. Reflection coefficients for incident plasma ions are measured using monoenergetic beams. Hydrogen trapping and permeation is studied in plasma simulators and atomic beams. Hydrogen release is typically studied using thermal desorption. These laboratory data provide the fundamental basis for developing physical models for the phenomena and are the standard to which the computational models are compared. 


\subsection{Models}

Given an incident particle flux and composition the TRIM code (or variants like VFTRIM) can be used to calculate the surface erosion yield. These codes have been thoroughly calibrated against the experimental data. The erosion yields are the input to codes such as WBC and REDEP that follow the eroded material in a background plasma and calculate redeposition and loss to the far field SOL. These codes rely on background plasma conditions that are either experimentally measured (rare) or calculated by an SOL simulation code such as UEDGE. In all cases the background plasma is considered to be constant in time, i.e., the eroded material does not alter the background plasma conditions. For transient events like ELMs or disruptions where the heat flux is large enough to cause melting of the material, the HEIGHTS code is used to simulate the melting and evaporation of the PFCs. This code also needs plasma conditions at some up stream location as a boundary condition, but it can calculate the impact of the eroded material on the plasma near the plate (e.g., radiative cooling). There are a variety of codes that treat impurity transport with a Monte Carlo method such as DIVIMP and ERO. Monte Carlo methods are also used to simulate neutral gas transport in complex geometries, e.g., DEGAS code. These codes all assume constant background plasma. In order to examine phenomena that are difficult to study experimentally or those that are very complex short duration interactions (radiation damage, chemical reactions, etc.), a variety of molecular dynamics (MD) models are used. These all depend on accurate knowledge of inter-atomic potential between material atoms and the incident species. MD models have been used to calculate reflection coefficients at energies too low for experiments.

\subsection{Development needs}

Since the amount of eroded material is only rarely small enough to not perturb the SOL plasma (both near the PFC and in general throughout the SOL), detailed understanding of the SOL, impurity transport, and hydrogen recycling will only be realized when all of the various codes are closely coupled and computing power is sufficiently large to permit iterative solution of the coupled models. This is presently done very crudely by exchange of data files. Integrated treatment of incident flux, impurity generation and transport is essential for understanding the SOL and impurity generation on long pulse devices and eventually having a predictive capability for new PFC materials.

\section{Atomic, molecular, and particle-surface processes (coordinated by D.R. Schultz)}

The edge plasma has been shown to play a determining role on core performance witness, for example, the direct effects of the application of carbonization, boronization, beryllium gettering, and lithium wall coating on the maximum attained performance of DIII-D, TFTR, and JET. In general, edge plasma conditions are the result of a selfconsistent interaction between it and the surrounding material surfaces. This interaction is mediated not only by direct plasma-surface interactions (PSI) but also by collisions between the plasma and neutral species resulting from PSI.

Thus, the edge plasma is dynamically affected by a significant range of atomic, molecular, and particle-surface interactions (AM\&PSI). These include, for example, 
influx of particles from wall erosion and from recycling, plasma energy and momentum transfer from elastic and inelastic collisions with atoms and molecules, and particle and charge state populations influenced by cycles of excitation, ionization, and recombination and from molecule formation and fragmentation. Therefore, theory, modeling, and simulation of the edge are crucially dependent on the existence of reliable AM\&PSI data and these data are a sine qua non for development of a predictive model of core performance.

Of equal importance, atomic collisions and consequent light emission also provide our experimental knowledge of edge plasma properties. Diagnostics such as those based on plasmas spectroscopies, CDD and CID camera and filterscope imaging, and charge exchange recombination spectroscopy using neutral beam injection are current examples. These means of observing and probing the edge reveal particle densities, ionization distributions, and ion temperatures, sense flows and rotations, and can gauge electric fields. AM\&PSI data are therefore also crucial for the operation and success of such diagnostics.

AM\&PSI processes also provide a means of actively controlling the edge plasma and are at the root of certain technological issues. For example, increasing edge radiative cooling via hydrogen or impurity gas puffing to ameliorate ELM-induced heat fluxes, demonstrating the feasibility of all metal (e.g., W) machines; understanding plasma conditioning (e.g., oxygen gettering by wall boronization, reduction of impurity influx, density control); providing enhanced recombination near the walls or in the divertor through molecular processes; forming a model for the cluster or dust formation resulting from wall processes; and developing a predictive model for detachment in divertors by understanding charge transfer and elastic scattering that catalyze enhanced temperature drop and neutral density increase.

Active communities of researchers are continually making new AM\&PSI measurements and computations, and much is known from the long development of the AM\&PSI fields. In fact periodic assessments of the status and priority needs for AM\&PSI data are made by various panels or working groups and an international network of atomic data centers (e.g. the IAEA A+M data center, the ORNL Controlled Fusion Atomic Data Center, the NIFS Data and Planning Center at Toki, and the Spectroscopic Data Center at NIST) have long been established to assist in the collection, evaluation, and dissemination of AM\&PSI data. However, significant shortcomings exist in a number of areas:

- While large bodies of high quality data exist, there are significant gaps and deficiencies regarding spectroscopy and collisional and physical/chemical behaviors of materials and species involved in present and next-generation plasma experiments,

- Communication of the present needs between the plasma science community and the AM\&PSI communities is not always timely, clear, and robust,

- Clear prioritization and support for remediation of AM\&PSI data needs is often lacking, 
- Efforts to systematically collect, evaluate, and disseminate AM\&PSI data exist but are often not sufficient,

- Significant development of atomic and molecular collision, PSI, and spectroscopic experimental facilities and computational codes is needed to be able to address present and future AM\&PSI data needs,

- Efforts to make AM\&PSI databases suitably comprehensive and formatted for use in modeling and simulation codes, or to be employed by diagnostics, is often not carried out well enough or uniformly enough, and

- Efforts to periodically re-evaluate and update AM\&PSI data are often lacking even though it is recognized that certain existing databases clearly need improvement.

Thus, a clear opportunity exists for the ECC to assist in improving the conduit for the needed communications and to spur the needed organizational actions. Such activity is crucial for the success of OFES's ongoing SciDAC and planned FSP programs, in support of diagnostics on existing US and international fusion devices and for smaller scale plasma experiments and simulators, for theory, modeling, and simulation of these experiments and for predictions of operational modes of devices such as ITER. For example, crucial for ITER is a full evaluation of the PSI properties of tungsten and carbon (e.g. studies of tritium retention and of tritium removal schemes, an improved understanding of chemical sputtering), simulation of gas puffing induced radiation in the edge, determination of the consequences of hydrocarbon molecule production at $\mathrm{CFC}$ surfaces, determination of spectroscopic and collisional properties of tungsten ions, etc., all requiring extensive new AM\&PSI studies and evaluations.

In summary, significant needs exist for coordinating the production, collection, evaluation, and dissemination of AM\&PSI data relevant to edge modeling and diagnostics. This should be a continuous process so that as models and diagnostic tools are employed and subsequently improved, the AM\&PSI databases are assessed and reevaluated. Close communication is required between the producers of AM\&PSI data, those that collect and evaluate it, and the edge plasma science community that uses it. Advanced computational methods for production of AM\&PSI data should be encouraged as well as experimental measurements that benchmark the data.

\section{Extension of Nonlinear Formulations to Edge Relevant Regimes} (coordinated by T.S. Hahm)

Nonlinear gyrokinetic formulations have provided the underlying theoretical foundations for remarkable recent advances in nonlinear gyrokinetic simulations of tokamak microturbulence as well as for analytic nonlinear theories of microturbulence. Both formulations and simulations traditionally focused on tokamak core turbulence in which the fluctuation amplitude is relatively small, i.e., the relative density fluctuation amplitude is less than one percent and the gradients in macroscopic parameters such as pressure are relatively mild with characteristic lengths on the order of a fraction of the 
minor radius. In the nonlinear gyrokinetic theory, there exist three expansion parameters; $\omega / \Omega_{\mathrm{ci}}, \rho_{\mathrm{i}} / \mathrm{L}_{\mathrm{B}}$, and $\delta \mathrm{f} / \mathrm{F}_{0} \sim \mathrm{e} \phi / \mathrm{T}_{\mathrm{e}}$. The early standard gyrokinetic theory assumes that all three parameters are comparable in formal ordering. This standard ordering is quite generic for core turbulence in which the spatio-temporal scale separation is clear, as stated above.

As experience in enhanced tokamak energy confinement accumulated with $\mathrm{H}$ mode operation, tokamak edge plasmas became hotter and less collisional. Therefore, traditional edge turbulence simulations based on Braginskii-type short mean free path fluid equations are not strictly applicable to leading present day tokamaks. Furthermore, in future devices, the standard fluid approximations will clearly break down. For instance, the mean free path at the top of the pedestal in ITER is about 40 times the connection length, assuming a density $10^{20} \mathrm{~m}^{-3}$, electron temperature of $3 \mathrm{keV}$, and $\mathrm{q}=3$.

For more accurate simulations of such collisionless edge plasmas, kinetic effects associated with long mean free path and large orbit sizes should be included properly.

In trying to apply the standard nonlinear gyrokinetic equations to edge turbulence, the following facts introduce challenges. Firstly, the relative fluctuation amplitudes in Lmode edge plasmas are typically on the order of 10 percent inside the last closed flux surface (LCFS), and can be even higher in the scrape off layer (SOL). Secondly,

after an $\mathrm{H}$-mode transition, an $\mathrm{E}_{\mathrm{r}}$ well is formed just inside the LCFS. The pressure gradient scale length and the radial electric field scale length in the edge $E_{r}$ well is on the order of the ion poloidal gyroradius,. Finally, not only in H-mode plasmas, but also in some L-mode plasmas, we have $\rho_{i} / L_{p}>L_{p} / R_{0}$ at the edge. Since $\rho_{i}$ is a typical unit for the radial scale of microturbulence, and $L_{p}$ is the length scale of a macroscopic quantity, we have the intriguing situation where one of the primary smallness parameters, $\rho_{\mathrm{i}} / \mathrm{L}_{\mathrm{p}}$, in the conventional nonlinear gyrokinetic formulation is greater than the ratio between two macroscopic scale lengths.

As more flexible and systematic modern symplectic perturbation theory was adopted for derivation of the nonlinear gyrokinetic equations, it was widely recognized that the standard nonlinear gyrokinetic ordering was not unique, and that one can develop nonlinear gyrokinetic equations in which some of the edge turbulence characteristics described above can be captured. While the motivations for such generalization were mostly due to the observation of strong ExB shear flows in core transport barriers and of self-generated zonal flows in simulations, progress has been very encouraging for the future development of edge relevant fully electromagnetic nonlinear gyrokinetic equations. Some of the key achievements to date are:

- Self-consistent inclusion of strong ExB flows in the electrostatic general geometry energy conserving nonlinear gyrokinetic Vlasov-Maxwell equations.

- Demonstration of the robustness of nonlinear electromagnetic gyrokinetic equations in the drift-kinetic regime with large amplitude, low frequency electrostatic potential fluctuations. 
- Wider recognition that the gyro-phase dependent information can be rigorously kept in the modern gyrokinetic derivation and can be used for calculation of relevant physical quantities.

With this current status in mind, extension of the existing fully electromagnetic nonlinear gyrokinetic equations to edge plasma turbulence in toroidal geometry capturing both large fluctuation amplitudes in L-mode and residual fluctuations in the presence of steep gradients in H-mode should be possible in the next five years. Generalized ordering should allow the poloidal ion gyroradius to be on the order of the radial scale length of $E_{r}$, $\mathrm{k}_{\text {perp }} \rho_{\mathrm{i}} \sim 1$, and $\mathrm{e} \phi / \mathrm{T} \sim \delta \mathrm{B} / \mathrm{B} \sim \rho_{\mathrm{i}} / \mathrm{L}_{\mathrm{p}}$ for large amplitude fluctuations.

The phase-space action variational Lie-perturbation theory will ensure that the conservation properties of the primitive set of equations will be preserved. In the derivation of a full $\mathrm{F}$ equation, it is not necessary to specify the form of $\mathrm{F}$. It is conceptually simpler to formulate gyrokinetic equations based on single particle orbits in terms of $E_{r}$ rather than in terms of mass flows. Thus, the form of the equation will be robust independent of $\mathrm{F}_{0}$, without assuming near-Maxwellian $\mathrm{F}_{0}$ nor requiring the use of neoclassical theory to determine the equilibrium $\mathrm{F}_{0}$.

Neoclassical equilibrium, i.e., distribution function in the absence of the turbulence, in the steep pressure gradient edge region can be calculated numerically as an input for turbulence simulations. A massively parallel, Monte-Carlo guiding center simulation could tabulate the distribution function in the 4D phase space. The main task here is to develop a parallel binary collision operator that is faithful to the Fokker-Planck operator, which is valid for arbitrary distribution function. The key assumption is that the ExB shearing rate is much smaller than the ion cyclotron frequency, so that the magnetic moment is still an invariant and guiding center concept is still valid.

While a fully kinetic treatment of the edge would be the most rigorous and reliable approach, it is even more challenging computationally and it is useful to have a parallel effort on a reduced fluid treatment of the edge, which would be faster or allow somewhat higher resolution. (The actual computational advantages might not be as large as one might first think, because fluid models of kinetic effects require evaluation of nonlocal conduction integrals, and core kinetic simulations have found convergence can be achieved with a relatively low number of velocity grid points using higher-order velocity integration methods.) Nevertheless, the two approaches have different computational characteristics and different ways of thinking about the physics and would provide an essential cross check on each other, particularly since there is a rigorous high collisionality limit in which the two approaches should be equivalent.

Because many actual edge plasmas of interest include regions where the mean free path is much longer than the parallel grid spacing and parallel gradients, standard fluid equations become inaccurate and it is important to include some kind of model of kinetic effects. Standard collisional fluid equations contain parallel transport terms of the form $q=-n \chi_{\|} \nabla_{\|} T$, where the parallel heat conduction $\chi_{\|} \sim \mathrm{v}_{\mathrm{t}}^{2} / v$ becomes unphysically large as the collision frequency, $v$, gets small. While a systematic derivation of fluid models of kinetic effects appropriate for the edge region has not yet been worked out, a first step to approximating kinetic corrections is to replace collisional parallel transport terms of the form $q=-n \chi_{\|} \nabla_{\|} T$, with a non-local heat flux, which in limit of small amplitude perturbations can be written in the form: 


$$
q(z)=-c_{1} n \mathrm{v}_{\mathrm{t}} \int_{0}^{\infty} d z^{\prime} \frac{T\left(z+z^{\prime}\right)-T\left(z-z^{\prime}\right)}{z^{\prime}} \frac{1}{1+c_{2} z^{\prime 2} / \lambda_{m f p}^{2}}
$$

where the integral could be truncated a distance $z$, along field lines of a few mean free paths $\lambda_{\text {mfp }}$ or a few parallel decorrelation lengths (whichever is shorter). The first term in the integrand can be interpreted as a kind of non-local averaged temperature gradient, and one can show that this expression reduces to the standard Braginskii collisional closure in the short mean free path limit, and also show that this is a multipole approximation to kinetic effects such as Landau damping in the long mean free path limit. Modifications of the form of this closure have been worked out by various people to better approximate certain neoclassical effects such as Rosenbluth-Hinton flows or bootstrap currents or magnetic pumping. Some of these modifications have only been done in the limit of mode frequencies small or large relative to the bounce-frequency, while it may be more challenging to be able to handle both limits simultaneously (which is important for the edge) in a single set of equations. Recent work indicates that it is possible to improve on the small-perturbation assumption used in the above non-local expression. Work could also be done to improve these formulations for intermediate collisionality regimes. Nevertheless, these are still approximations, and it will be useful to also have fully kinetic approaches to compare with.

For more accurate kinetic effects, it is better to reformulate the fluid equations to keep more fluid moments, at least using separate evolution equations for $\mathrm{p}_{\|}$and $\mathrm{p}_{\perp}$ (whose dynamics differs from $\mathrm{p}_{\|}$because of $\mu$ conservation). This avoids many of the complications of the stress tensor and gyroviscous cancellations and allows the retention of collisional exchange between the perpendicular and parallel part of the energy as needed. Separate evolution equations for $\mathrm{q}_{\|}$and $\mathrm{q}_{\perp}$ can also significantly improve accuracy, as they naturally allow for non-zero but non-dissipative heat fluxes that occur in toroidal equilibria.

\section{Integrated H-mode Edge Modeling (coordinated by G. Bateman)}

Integrated modeling of the H-mode pedestal, ELMs, scrape-off-layer (SOL) and plasmawall interactions provides a self-consistent way to bring together all of the strongly interacting physical phenomena at the edge of $\mathrm{H}$-mode plasmas and provides the boundary conditions needed for whole device simulations. It is clear that integrated edge simulation capability is vital for ITER and burning plasma experiments. The edge pedestal in H-mode discharges has a significant impact on plasma confinement and transport through the edge region impacts the ability to handle power, helium exhaust, and particle fueling. It is known that predictive simulations of the core temperature and density profiles depend sensitively on the conditions at the edge of the plasma, particularly in H-mode tokamak discharges. Integrated simulations are needed for accurate predictions of the performance of burning plasma experiments such as ITER.

At the present time, relatively simple models are used within integrated modeling simulations to predict the height, width, and shape of the pedestal at the edge of H-mode discharges, as well as the frequency of ELMs and changes in the edge profiles produced by periodic ELM crashes. Simulations reproduce many of the trends observed in the experimental data as a function of plasma density, heating power, triangularity, isotope 
mass and other plasma parameters. There has been some calibration of the simulations with experimental data, particularly for the pedestal height and ELM frequency. Most of the integrated modeling simulations span the region within closed magnetic surfaces, from the center of the plasma to the separatrix. Recently, most of the effort has been concentrated on H-mode discharges with type 1 ELMs. In some cases, the output profiles from integrated simulations have been used as the input to more specialized computations, such as full-featured MHD instability codes.

It is clear that more sophisticated models are needed for use within integrated simulations in order to compute the following physical phenomena: (1) the changes in the edge plasma profiles that are produced by each ELM crash, (2) the extent to which different channels of transport are suppressed in the pedestal (ie, electron thermal vs ion thermal), (3) the outer boundary conditions for the pedestal (inner boundary conditions for the scrape-off-layer) and the influx of neutrals into the plasma, (4) the power and particles lost to the divertor and first wall, and (5) the effect of the vertical asymmetry on the $\mathrm{L}$ to $\mathrm{H}$-mode transition and the behavior of the subsequent $\mathrm{H}$-mode plasma. In addition, the models used within integrated simulations must be extended to apply to different types of ELMs, as well as ELM free discharges and plasmas with ergodic divertors.

Simulations of the detailed structure of ELM crashes are currently being carried out using non-linear macroscopic instability (extended MHD) codes. For integrated simulations, models are needed to determine changes in the edge profiles of the temperatures, densities, and the current density that are produced by each ELM crash. It is particularly challenging to develop a model for the change in the current density profile, since the burn-off of current density in the pedestal is probably caused by many fine-scaled magnetic reconnection events during the ELM crash. The required ELM crash model must also compute the radial extent of the changes in the edge profiles and the amount of plasma energy and particles lost to the wall during each ELM crash as a function of edge conditions.

Kinetic turbulence and neoclassical simulations are now being applied to the steep pressure gradient region of the pedestal in order to develop models for the different channels of transport (electron and ion thermal, hydrogenic and impurity particle transport) through the pedestal. The simulation of transport through the pedestal is particularly challenging for at least four reasons: (1) the gradient scale lengths are comparable to the widths of the banana orbits and turbulent convection cells; (2) there is a strong flow shear that reduces, but does not completely eliminate, anomalous transport in the pedestal; (3) the plasma geometry near the separatrix is complicated; and

(4) particle distribution functions are likely to be distorted far from a Maxwellian distribution. In spite of these obstacles, kinetic simulations are likely to produce significantly better transport models within the next five years.

During each ELM crash, roughly $10 \%$ of the plasma is lost to the divertor and first wall. Most of the lost ions return rapidly to the plasma as neutral atoms. It is expected that most of the returning neutral atoms charge exchange many times as they pass through the scrape-off-layer and into the confined plasma. It is necessary to model the returning neutral atoms reasonably accurately, since they produce a source of plasma ions that is strongly peaked in and around the pedestal region, and this source of ions is 
likely to have a strong influence on the resulting edge plasma density profiles. In addition, the plasma temperature at the base of the pedestal is expected to vary rapidly during each ELM crash. In order to model these physical phenomena and to predict quantitatively accurate results, it will probably be necessary to couple a two-dimensional scrape-off-layer code, a plasma-wall interaction code, and a neutrals code together within a whole device integrated modeling code.

Experimental evidence indicates that ELM crashes have a complicated structure in which filaments of plasma break off and move to the outboard wall while most of the heat is conducted along each filament to the divertor. The models used for ELM crashes within integrated simulations will have to include the effects of this complex structure in order to make accurate predictions of the spatial extent and time dependence of the power deposition on the divertor plates and the recycling of particles at the edge of the plasma. These predictions are particularly important for burning plasma experiments such as ITER.

It is known that the spatial relationships between the nearest separatrix X-point and the ion magnetic drifts have a strong effect on the heating power needed for the $\mathrm{L}$ to $\mathrm{H}$-mode transition and on the quality of confinement in the resulting H-mode plasma. It is clear that this vertically asymmetric effect must be included to produce an accurate model of H-mode plasmas. Theoretical explanations of the vertical asymmetry effect have not yet been included within integrated modeling codes.

Finally, the models used within integrated simulations must be extended to predict the different types of ELMs as well as the ELM-free discharges that are observed under some conditions in experiments. Simulating this diversity of physical phenomena can help to validate the models used in the integrated codes.

\section{Priorities during the next five years:}

Integrated edge modeling must incorporate the following elements: micro-turbulence, intermittent meso-scale physics ("blobs"), formation of edge transport barrier (L-H transition), ELMs, bootstrap current, neoclassical transport, dust generation and transport, neutral-particle and impurity transport, and wall erosion processes. Integrated edge modeling brings together very different physical processes such as flow shear stabilization of turbulence, large scale instabilities that produce ELM crashes, formation and motion of plasma blobs, and the strong interaction between the plasma, neutrals, and the first wall. The edge modeling spans a wide range of time scales from the microsecond time scale of turbulence to the millisecond time scale of ELM cycles to the multiple second time scales of wall erosion.

During the next five years, the priorities for integrated edge modeling include:

- Integrate accurate models for MHD instabilities that trigger ELM crashes, ranging from short-wavelength ballooning modes to long-wavelength kink-peeling modes.

- Develop a more accurate simplified model for the non-linear evolution of each ELM crash. In particular, integrated edge modeling codes require a model for the changes that occur in the edge current density, temperature and density profiles as a result of each kind of ELM crash. 
- More accurate models based on first-principles simulations are needed for neoclassical transport and the flow shear suppression of turbulent transport within the pedestal, including the effects of (1) steep gradients, (2) large flow shear in the narrow region of the pedestal, (3) complex geometry including the separatrix, and (4) non-Maxwellian particle distributions.

- Integrate simulation of the scrape-off-layer (SOL) together with the simulation of the $\mathrm{H}$-mode pedestal and ELM crashes. In particular, the SOL simulation is needed to compute the neutral influx and the outer boundary condition of the pedestal as a function of time throughout the ELM cycle.

- Integrate the response and erosion of the first wall and the divertor.

- Integrate models for the effects of toroidal asymmetry and error magnetic fields on edge transport and ELM crashes. Experimentally, it is observed that ergodic fields at the edge of the plasma have a large impact on ELM frequency.

- Incorporate the effect of the vertical asymmetry on the L to H-mode transition and the behavior of the subsequent $\mathrm{H}$-mode plasma.

- Validate the edge integrated modeling by comparing the results of simulations against experimental data under a wide variety of conditions, including different types of ELMs as well as ELM-free discharges.

\section{Computational Issues Related to Simulation of Edge Plasmas (coordinated by D.D. Schnack)}

What Problem Are We Trying to Solve?

The edge presents one of the most challenging problems in computational physics. Many disparate physical processes play a role in determining this evolution. The traditional separation of equilibrium, stability, and transport no longer applies. Spatial scales vary over 10 orders of magnitude, from atomic and molecular $\left(\sim 10^{-10} \mathrm{~m}\right)$ to macroscopic $(\sim 1 \mathrm{~m})$, and time scales vary from over 6 orders of magnitude, from ion gyration $\left(\sim 10^{-8} \mathrm{sec}\right)$ to macroscopic evolution $\left(10^{-2} \mathrm{sec}\right)$. The plasma interacts with the wall and recycling occurs. Small scale processes affect the long wavelength dynamics, and the details of geometry are important. The mean plasma parameters can vary significantly on short spatial scales, and the amplitude of time dependent fluctuations can approach or exceed the mean values. There is no clear separation of scales as is often the case in hydrodynamic or MHD turbulence. It is difficult to find a "reduced" model.

At the present time is no clear consensus within the edge community on the problem to be targeted by computations. Candidates are 1) nonlinear ELM development, including advection through the scrape-off layer and power deposition on the wall;2) L$\mathrm{H}$ transition; 3) details of the fluid turbulence in the edge; 4) full ELM cycle, including profile relaxation, pedestal rebuild, and neutral influx; and, 5) fully kinetic (5-D) calculations of edge transport. Each of these problems emphasizes different time and space scales, and likely dictates a different computational approach. Informed decisions on algorithm design cannot be made in the present environment. It is important for the community to reach consensus on the specifics of the target problem. 


\section{What Equations Should We Solve?}

The fundamental dynamics of this plasma is described by a kinetic equation for each species, which relates the change in the total time derivative of the distribution function to collisional interactions. This model requires 5 independent variables ( 3 spatial coordinates and 2 phase space coordinates) plus time. In principle, this model

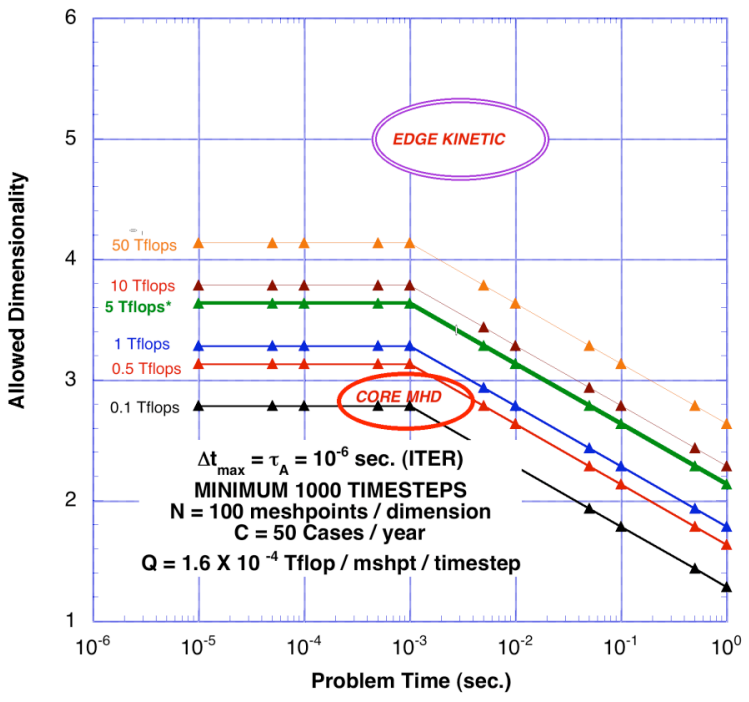
contains all the information about the evolution of the plasma. Constraints on modeling macroscopic, low frequency dynamics can be determined by balancing code performance (Tflop per meshpoint per timestep) and problem requirements (dimensionality, resolution, problem time, time step, number of cases per year) with the available supercomputing cycles per year (Tflop). The results for the case of core plasma simulation with an existing extended MHD code are shown in the figure. Problem dimensionality is constrained by both problem time and delivered computing power. As indicated, relevant 3-dimensional MHD calculations can be can be carried out for several msec problem time with presently available resources. Detailed implications for edge modeling await estimates of performance data for existing and planned edge fluid and kinetic codes. However, from the present data it seems clear that kinetic calculations with 5 dimensions for 10 or more msec problem time require more resources than can be presently envisioned. Thus, meaningful simulations of edge dynamics must employ a 3-dimensional fluid model.

The simplest fluid model is MHD. The linear stability of ELMs can be studied within the MHD framework. However, MHD alone cannot describe the important subgrid scale kinetic physics that affects the long wavelength motions, and extensions of these equations through closures of the underlying kinetic equations must be employed. As yet there is no agreement within the community as to the precise form of the equations to be solved. Proposed models range from reduced Braginskii ${ }^{1}$ to more complicated closure schemes that incorporate higher order moments of the distribution function ${ }^{2-5}$. Some employ the drift ordering, with the underlying assumptions of slow deviations from force balance. This may be appropriate for turbulence studies, but it is not clear that it accurately incorporates the time scales required for nonlinear simulation of ELMs (for example). Since the choice of equations has implications for computations, it is important that there be a resolution of this issue.

How Should We Solve Them?

Whatever the equations to be solved, they will be temporally stiff, and that dictates some sort of implicit approach to time discretization. The edge plasma presents two types of stiff problems. The first concerns turbulence, in which the fundamental stiffness comes from nonlinear dynamics. All terms in the equations play a role, and a fully implicit approach based on a Newton-Krylov scheme is reasonable. The second concerns instability, in which the fundamental stiffness comes from the large range of the 
eigenvalues of the underlying linear problem. Here the semi-implicit approach is very efficient. While the drift ordering eliminates the numerically troublesome whistler branch, the kinetic Alfvén wave, which is also dispersive, remains. This may require special attention. The self-consistent ELM/pedestal problem involves both processes, and it is not clear which computational approach will be most effective. This issue needs further consideration. Both approaches require efficient, parallel linear solver software. The proper preconditioning can also greatly affect algorithm performance.

The primary issue for spatial discretization is the extreme anisotropy introduced by the strong magnetic field. This affects the turbulence, the transport, and the faster instability dynamics. Failure to model this property accurately can lead to spectral pollution and anomalous cross-field transport. Present approaches ${ }^{1}$ use a ballooning coordinate system that allows simplification of parallel and perpendicular derivatives. Recently high order finite elements coupled with flux-aligned grids have proven effective in the accurate simulation of core plasmas ${ }^{6}$. This approach should be considered for the edge as well.

Any code developed to solve the fluid equations should be modular, readable, and accessible to modification a wide variety of users. This dictates F90 as the language of choice. This code can form the basis of an integrated computational program.

Further algorithm design cannot proceed until there is consensus on the target physics problem, and the form of the equations, to be solved.

\section{Verification and Validation (coordinated by D.P. Stotler)}

The basic purposes of verification and validation $(\mathrm{V} \& \mathrm{~V})$ are to enable us to interpret code calculations with a level of rigor and error analysis commensurate with that found in good theoretical and experimental papers. Verification and validation are essential if simulations are to truly become the "third leg" of scientific research and if simulation results are going to play a larger role in funding decisions and planning for future experiments. A record of the $\mathrm{V} \& \mathrm{~V}$ activities performed on a particular code should be part of that code's publicly accessible documentation so that it can be examined by the code's users and by reviewers of scientific papers featuring results produced by that code.

The computational fluid dynamics (CFD) community has developed, and is continuing to develop, elaborate techniques for verifying and validating their codes. These are well documented in the literature. The Accelerated Strategic Computing Initiative (ASCI) has also recognized the need for $\mathrm{V} \& \mathrm{~V}$ and has made it a centerpiece of their code development process. The fusion edge plasma community can use their efforts as starting points for our own V\&V program.

"Verification", loosely described, consists of ensuring that a code correctly solves the equations upon which it is based. This process can be broken into two components: demonstrating that the code is free of mistakes, and establishing the accuracy with which the code solves a given problem.

The need for the first task becomes clear when one learns that even commercial Fortran codes have, on average, 12 faults per 1000 lines of code. Software quality engineering (SQE) tools can help with this. One example is "static analysis" in which the structure and syntax of the code is examined without executing it, similar to what a very 
strict compiler would do. Another is "regression testing". This refers to the development of a suite of test cases, each exercising a particular code capability, which can be run after each modification to the code. Unexpected deviations from the baseline results are investigated to see if they are the result of newly introduced bugs. Although the actual running of the test suite can be automated, significant programming effort must be devoted to assembling and maintaining the test cases.

The second half of verification, verification of calculations, involves demonstrating that the code solves a particular problem with the expected level of accuracy. As an example, a CFD codes may be susceptible to errors caused by inadequate spatial discretization, inadequate temporal discretization, or lack of iterative convergence; these same sources of error can arise in edge plasma modeling codes. The error can be quantified by comparing the code's result with a solution known to be of higher accuracy. CFD codes are not only required to converge to that solution as the grid is refined, but to converge at a rate that matches expectations.

The Method of Manufactured Solutions provides a valuable alternative when nontrivial solutions to the equations are unavailable. One begins by concocting a solution, perhaps nonphysical, with sufficient structure to exercise all of the terms in the equations but with enough smoothness that the required derivatives can be readily computed and evaluated. The sources and boundary conditions corresponding to this solution are determined by simply substituting it into the equations. These are in turn used as input to the code. The code's results can then be quantitatively compared with the expected result. Of course, this procedure is facilitated by having a code that can handle arbitrary sources and boundary conditions.

Some problems place additional, physical requirements on the spatial and temporal discretization used. Namely, the simulated dynamics must reproduce that expected from the underlying equations. For example, an edge plasma turbulence simulation must be capable of handling all relevant eigenmodes. To this end, all physical scales from $\rho_{\mathrm{s}}$ (ion Larmor radius computed with the electron temperature) to $L_{\perp}$ (gradient scale length perpendicular to the magnetic field) must be resolved in both directions perpendicular to the magnetic field line. Moreover, if constant (in space) parameters are used, the radial size of the computational domain can be no larger than $\mathrm{L}_{\perp}$. Parallel transit times must be resolved, leading to similar restrictions on the temporal discretization.

"Validation" is a multi-step process. At each step, code results are compared with measurements from one or more experiments. Carefully evaluated error bars on both simulation and experimental data provide quantitative bounds for the acceptable level of disagreement; the phrase "rough agreement" has no place in this context. Again, the CFD community's experience can provide guidance in the construction of "validation metrics" that serve as the basis for a rigorous statistical comparison.

The first step is to test individual components of the problem against simple, thoroughly diagnosed experiments specifically designed for that component, e.g., a tabletop or linear plasma device. Even though such experiments will miss significant pieces of physics that arise only in the full, integrated system (i.e., the tokamak divertor), they are rarely too simple. One invariably learns something. Even demonstrating that the 
physics studied in the simple system is qualitatively different from that in the full system (if that's what is concluded) constitutes an advance in the overall understanding.

The second step is a "benchmark" test involving two or more physics components of the problem. The corresponding experiment will again have been designed to facilitate a high quality comparison, utilizing a simple geometry, permitting simplifying assumptions or easing diagnostic measurements. The third step, "subsystem" tests, again utilizes only a subset of the full physics components of the problem, but the corresponding experiments are carried out in a situation that is closely related to that of the end objective. An example might be simulation of a tokamak divertor plasma operated in steady state at low density and high temperature. The final step is a test of the fully integrated model against a correspondingly unconstrained experiment. Of course, at this level the experimental data may be harder to come by or of lower quality than those utilized at the other levels. In complicated systems, such as the fusion edge plasma, developing a viable validation hierarchy can become a scientific endeavor in its own right.

Verification and validation are related to, but not synonymous with, the more familiar concepts of code comparison, also known as "code benchmarking", and "code calibration". A "benchmarking" of two or more codes against each other can contribute to the verification of those codes. If one of those codes has been validated, it can be used, in lieu of an experiment, to generate data suitable for validating the second code. "Code calibration" refers to the process of adjusting a simulation model's parameters to optimize agreement with experimental data. This is appropriate in cases where the simulation model and / or experimental data are not sufficiently well developed to permit rigorous validation and can be viewed as a precursor step to validation.

Specific tasks (in order of decreasing priority) that the fusion edge community and this Committee in particular, could undertake in the next five years to nurture a strong $\mathrm{V} \& \mathrm{~V}$ program include:

- Form a subcommittee to discuss in more detail the extent to which existing codes have been verified and make recommendations on further action.

- Establish a forum for presentation, and perhaps publication, of V\&V results. By doing so, we will help institutions recognize and reward $V \& V$ activities as essential for the long-term objectives of the program.

- Look into the use of available SQE tools for static analysis of existing codes and make a corresponding recommendation to the community.

- Collect and distribute, (e.g., via the ECC web site) solutions suitable for use in verification. These could include physically realistic solutions as well as "manufactured" ones. Physical constraints on spatial and temporal discretization, such as those noted above for edge plasma turbulence, could be list here as well.

- Encourage public documentation, via links on the ECC web site, of the V\&V of the principal edge codes.

- Coordinate and prioritize the development of simplified benchmark experiment concepts. Substantially greater expense and effort will need to be devoted to 
generating experimental data suitable for validation. Given the current goaloriented operating procedures for the major devices, cannot expect much help from them in obtaining such data without pressure from DoE, etc. We could start by putting forth some concrete examples of needed data. This objective strongly overlaps with those of the "Partnerships of theorists / modelers with experimentalists" topic.

\section{Modeling Partnerships with Experimentalists and International Programs (coordinated by J.L. Terry)}

\subsection{Status Summary}

Since the edge encompasses such diverse areas (i.e. pedestal, SOL, and material surfaces of the PFCs), the status of the each area's interaction or partnership with experiment is varied. Nonetheless, applicable to each area are the following general observations:

- We have had fruitful collaborations between edge modeling and experiment.

- Those collaborations of long duration and with longer-term goals have been the most successful. Typically these longer-term collaborations have had a scientist (either from the modeling or the experimental side) whose job description was in part to interface between the code and the experiment. Without such an assignment (and associated funding), the collaborations have tended to be informal and short term.

- Both the models and the experimental techniques have rapidly been getting more sophisticated, with the quality of data from each side improving. Nonetheless, comparisons with basic experiments are lacking in many cases.

- The frequency and quality of comparisons between modeling and experiment have increased. The results have so far been mixed. It is safe to say however that a satisfactory, predictive capability for the key research questions has not been achieved.

We note that each "edge" area has been addressed by different modeling efforts and that these are not well integrated at the present time. (An example of a well integrated, albiet very ambitious, simulation would be the time evolution of an ELM crash-and-recovery cycle, including description of the crash trigger, the heat and particle propagation through the SOL to the divertor and wall, material effects on the PFC, the pedestal re-establishment, etc.) It is also generally true that different groups are involved within each area, with a less than desirable degree of overlap and interaction among groups. At present the framework for modeling integration as well as for modeling/experiment integration is provided mainly by periodic meetings (TTF Conference, PSI Conference, ITPA meetings, H-mode Workshops) and by working groups (ITPA, IPPA). There is also general agreement that without additional funding or a shift in personnel allocation there is little chance to increase the rate of progress being made in area of modeling/experiment comparisons.

\subsection{5-Year "Roadmap"}

If we propose as an ultimate goal an integrated predictive capability for the edge with a small number of measurable inputs, then it is worthwhile to consider what steps we can 
take in the area of modeling/experimental partnerships within the next 5 years to approach that goal. We note that this issue is not unique to the edge. The same situation exists for comparison of core modeling with experiment. In the case of the core, an effort is being coordinated through TTF. We should be involved with and pay attention to that effort, copying what works. A suggested (and evolving) list of desirable initiatives that relate to modeling/experimental partnerships is:

- Create a short prioritized list of modeling/experimental comparisons that can be made. This should include some of the simplest and most basic experiments. If the sophisticated codes cannot reproduce the basic experiments, then there is no reason to believe "verifications" in the more complex comparisons The list should also include comparisons that may disagree significantly, so as to point out the needs for further refinement in both modeling and experiment. We recognize in this regard that the realities of funding and publication make it difficult to present those cases in which one's code doesn't work. A preliminary listing and prioritization of desired comparisons appears at the end of this document.

- Facilitate comparisons between modeling and experiments. This would include:

o Providing an environment within the community in which it is the norm for experimental data to be compared with modeling and vice versa. This involves reinforcement (via invited talks, recognition, etc.) of those people and cases that do so;

o Providing an environment within the community in which it is the norm to provide quantitative assessment of errors in both simulation and experiment;

- Striving to make the codes easy to use. This includes making the codes easy to run and making preparation of input data as easy as possible. If only the code-developer can run the code, its use will always be sporadic. In instances where "easy-to-use" means loss of believable results, we should foster the establishment and long term funding of effective teams of theorists/code-developers and shot-simulation experts.

- Creation of a "clearing house" for the dissemination of standard and tested analysis codes; for example, possible use of W. Nevins' GKV code as a standard for fluctuation or turbulence analysis.

- Promotion of a standardization of data transfer format; this would facilitate "post-processing" of a given simulation by people other than the person or group who actually runs the simulation; it would also facilitate use of experimental data by modelers;

- Providing a public listing of available modeling codes with a brief description of applicability, inputs, and, most importantly, contacts;

- Providing a public listing summarizing available experimental data with a brief description of measurement, plasma conditions, and, most importantly, contacts;

While the above list treats collaborations between modelers and US and international experimentalists on an equal footing, there is the special case of 
partnerships between US modelers and their international modeling counterparts. In this area it is desirable to consider benchmarking among comparable US/international codes. At present benchmarking of the international codes is being pushed by the SOL/diverter ITPA. We urge examination of the ECC Summary on the topic of Verification and Validation in this regard.

12.2. Prioritized list of modeling/experimental comparisons

Prioritization of issues has been into three categories: very high ( $\mathrm{VH})$ for significant progress in the next 5 years, high $(\mathrm{H})$, or interesting (I). A listing follows:

\section{Very high priority:}

- Edge Pedestal Structure (between ELMs) - of course this requires a viable model for pedestal transport

- Are GAMs/Zonal flows important in pedestal?

- Can the experiment distinguish between shear stabilization and electrostatic confinement?

$\circ$ Effects of the X-point effects

- Experimental tests focusing upon key physics should be devised for these issues, including experiments on simple laboratory devices to test isolated pieces of the physics, experiments on university-scale tokamaks for detailed turbulence measurements, and experiments on national and international tokamaks to validate key results.

- Tritium trapping, erosion, and migration

- Full dynamics of the ELM crash event - in addition, this would certainly provide a worthy subject for the 2005/06 OFES Theory milestone of simulating nonlinear plasma edge phenomena using extended MHD codes.

- Particle accounting in pedestal and SOL - this will require understanding of the next $\mathrm{VH}$ rated comparison, i.e.,

- SOL turbulence and transport

- Connection between intermittent, convective blob transport and density limits

- Structure of SOL turbulence

- Poloidal distribution of SOL turbulence

- Effects of primary and secondary separatrix on SOL turbulence

- Identify specific measurable quantities and experimental parameter scalings that will test the underlying physics of nonlinear simulations of turbulence and transport

- Role of neutrals in edge turbulence

$\circ$ Effects of the X-point

\section{High priority}

- Edge flows - Can we measure and model them?

- L-H transition dynamics

- Role of Reynolds stress in L-H transition

- Is L-H Transition due to 1) shear flow stabilization, 2) stabilization of peeling modes, or 3) stabilization of drift Alfven modes? 
- Is L-H transition a bifurcation or not?

- Impurity transport from source through SOL

- Is there compelling evidence of need for kinetic description in pedestal, in SOL? (The answer impacts crucially the effort spent on developing kinetic codes for the edge.)

- Understand non-Type I ELM Edge fluctuations e.g. Quasi-Coherent Mode, EdgeHarmonic Oscillation

\section{Intermediate priority}

- Ion energy threshold for chemical sputtering of C

- Can we measure and model the sheath transmission coefficient?

- L-H thresholds (why are they not constant?)

- Role of dust in plasmas

- Can actual surface (roughness, composition, etc.) ever be accurately modeled? 